\title{
Development of a Fast and Facile Analytical Approach to Quantify Radiometabolites in Human Plasma Samples Using Ultra High Performance Liquid Chromatography
}

\author{
Cécile Malherbe ${ }^{1,2}$, Rudy Bidault ${ }^{1}$, Claude Netter ${ }^{3}$, Denis Guilloteau ${ }^{1,4,5}$, \\ Johnny Vercouillie ${ }^{1,5^{*}}$, Nicolas Arlicot ${ }^{1,2,5^{*}}$ \\ ${ }^{1}$ UMR 1253, iBrain, Université de Tours, Inserm, Tours, France \\ ${ }^{2} \mathrm{CHRU}$ de Tours, Unité de Radiopharmacie, Tours, France \\ ${ }^{3}$ Thermo Fisher Scientific, Waltham, MA, USA \\ ${ }^{4} \mathrm{CHRU}$ de Tours, Service de Médecine Nucléaire in Vitro, Tours, France \\ ${ }^{5}$ INSERM CIC 1415, University Hospital, Tours, France \\ Email: "vercouillie@univ-tours.fr
}

How to cite this paper: Malherbe, C., Bidault, R., Netter, C., Guilloteau, D., Vercouillie, J. and Arlicot, N. (2019) Development of a Fast and Facile Analytical Approach to Quantify Radiometabolites in Human Plasma Samples Using Ultra High Performance Liquid Chromatography. American Journal of Analytical Chemistry, 10, 185-201. https://doi.org/10.4236/ajac.2019.105016

Received: March 12, 2019

Accepted: May 7, 2019

Published: May 10, 2019

Copyright $\odot 2019$ by author(s) and Scientific Research Publishing Inc. This work is licensed under the Creative Commons Attribution International License (CC BY 4.0).

http://creativecommons.org/licenses/by/4.0/

\begin{abstract}
Introduction: Conventional metabolite analyses often require manual sample preparation, generating variability of measurements. This study describes a new method to quantify radiometabolites in blood, combining ultra high performance liquid chromatography (UHPLC) and turbulent flow chromatography, an alternative fully automated process allowing analyte's extraction. Methods: A new radiotracer for dopamine transporter imaging, namely LBT-999, was used to demonstrate the method's robustness. Matrix effect, Turboflow column loading, linearity, specificity and precision were evaluated with in vitro samples of LBT-999 in human plasma. Radiodetector sensitivity and preliminary evaluation were respectively determined by analysis of calibrated samples of $\left[{ }^{18} \mathrm{~F}\right] \mathrm{LBT}-999$ and blood samples from 4 healthy subjects injected with $\left[{ }^{18} \mathrm{~F}\right] \mathrm{LBT}-999$, withdrawn at $5,15,30$ and $45 \mathrm{~min}$ pi. Results: With three sequential loadings $(3 \times 100 \mu \mathrm{L})$ of the Turboflow column, mean coefficients of variation were $1 \%$, below $2 \%, 2 \%$ and $30.9 \%$ for matrix effect, specificity, repeatability and intermediate precision, respectively. Correlation coefficients for linearity were superior to 0.97 . Limits of detection and quantification of the radiodetector were fixed at 3 and $9 \mathrm{c} / \mathrm{s}$. Retention times for $\left[{ }^{18} \mathrm{~F}\right] \mathrm{LBT}-999$ and the two radiometabolites detected by radio-UHPLC were 6.5, 4.8 and $9.6 \mathrm{~min}$. Forty-five min after the injection, parent fraction was still predominant with $57.8 \% \pm 25 \%$ of the total radioactivity. Conclusions: An innovative approach, allying UHPLC and Turboflow column, was developed
\end{abstract}


and its sensitivity, linearity, specificity and repeatability validated. Preliminary results of the clinical trial are in accordance with literature data, demonstrating its efficiency in radiometabolites quantification.

\section{Keywords}

Radiometabolite, Ultra High Performance Liquid Chromatography, Turboflow, Method Validation, $\left[{ }^{18} \mathrm{~F}\right] \mathrm{LBT}-999$

\section{Introduction}

Positon Emission Tomography (PET) is a molecular imaging technique used in nuclear medicine, to visualize, characterize and measure biological processes at the molecular and cellular levels [1] allowing for both anatomical and functional imaging [2]. PET explorations, prescribed for diagnosis in stratified medicine approaches [3] or therapy purposes, rely on the use of radiopharmaceuticals. These drugs, labelled with $\beta$-emitter isotope like fluorine-18 or carbon-11, are highly selective of the targeted biomarker, which imparts its molecular specificity to the PET exploration.

PET images do not only give access to qualitative images, but also to quantitative values of tracer's uptake by the brain, which generally requires the determination of the input function, the arterial blood activity, to deduce pharmacokinetic parameters [4] [5]. Thus, the density of a molecular target can be apprehended in a specific region. However, like any other drug, radiopharmaceuticals are metabolized in vivo, leading to different chemical structures carrying the radioisotope that PET cameras won't be able to discriminate [6]. These various molecular sources of $\beta$-emission may hamper the accurate quantification of PET measurement.

Metabolism is the physiological way to eliminate a substance from tissues and bloodstream; drugs are degraded into small fragments by physicochemical transformations [7] operated by enzymes, mostly in the liver, the blood or the brain [8]. Hydrolysis of the bond between the radionuclide and the vector is a common problem in in vivo transformation of radiopharmaceuticals (defluorination, dealkylation ...) because free radionuclides may jeopardize images' interpretation due to their tropism for specific tissues or organs. The high uptake of fluorine-18 $\left({ }^{18} \mathrm{~F}\right)$ by bones and particularly the skull, may generate an important partial volume effect and thus affects PET spatial resolution and images quantification [6]. Metabolism can also result in fragments still carrying radioisotopes, called radiometabolites, which can be challenging in two ways. Radiometabolization may preserve affinity of the metabolite for the target, which can interfere with the parent molecule uptake by competition phenomena or generate noise, depending on lipophilicity of the metabolite and ability to cross the blood-brain barrier (BBB), to bind to other receptors, aggregates or enzymes. This is particularly challenging in brain PET imaging, where the quantification of radiophar- 
maceuticals' uptake directly correlates with neuronal density. It is then crucial to characterize brain ingress of troublesome radiometabolites, in order to apply correct quantification parameters. Metabolism studies are essential for the complete characterization of a radiopharmaceutical drug, and are a key step in the development process for clinical use.

Historically, thin layer chromatography (TLC) was the first technique used in order to separate radioactive compounds [9]. TLC separation is due to interactions of the compounds of interest, depending on its relative distribution between two non-miscible phases; a solid stationary phase consisting of an adsorbent coated on a plate, foil or sheet, and a liquid mobile phase which is drawn up the plate by capillary action. This approach is easy to implement, rapid, cheap and all the radioactivity of the deposit will be detectable without any loss of information. TLC is to date a relevant method routinely used in radiopharmacy departments, for daily determination of the radiochemical purity of radiopharmaceuticals. However, TLC is mostly a qualitative technique, since peak resolution, precision and repeatability are limited. Because of these limitations, TLC is not suitable for metabolism studies.

To date, the reference method for radiometabolites studies is High Performance Liquid Chromatography (HPLC), coupled to radioactivity detection. Analyses are highly reproducible and allow the quantification of narrow peaks or even rider or shoulder peaks, which is not possible with TLC. However, blood samples cannot be directly injected into the HPLC system; this technique requires a mostly manual sample preparation step for both matrix removal and decrease in risk of column clogging. Therefore, the blood is centrifuged, the obtained plasma is mixed with an organic solvent to precipitate plasmatic proteins, and then centrifuged again [10]. The resulting supernatant, containing the molecules of interest, is injected in the HPLC system. This relatively long preparation process combined with the short half-lives (110 min for fluorine-18, $20 \mathrm{~min}$ for carbon-11) of PET radiotracers may cause a loss of information, especially for the late time points of PET imaging protocols. Furthermore, the multistep blood extraction could lead to losses or modifications of information, inducing a bias in analysis repeatability.

Thus, to overcome these limitations, we applied an alternative approach to explore radiopharmaceuticals metabolism over time, allowing a more efficient sample preparation with shorter analysis time, and better compounds separation. An online sample preparation system based on turbulent flow chromatography (TFC) was first used to extract the molecule of interest from the matrix, the latest being eliminated. The extracted sample was then injected into the analytical column of an Ultra HPLC (UHPLC) system for chromatographic separation with radio and UV detection. The tracer used in the accomplishment of this work was a new PET radiopharmaceutical: (8-((E)-4-fluoro-but-2-enyl)-3-beta- $p$-tolyl-8aza-bicyclo[3.2.1] octane-2-beta-carboxylicacid methyl ester) or $\left[{ }^{18} \mathrm{~F}\right] \mathrm{LBT}-999$ (Figure 1), a tropane derivative with high affinity for the Dopamine Transporter 


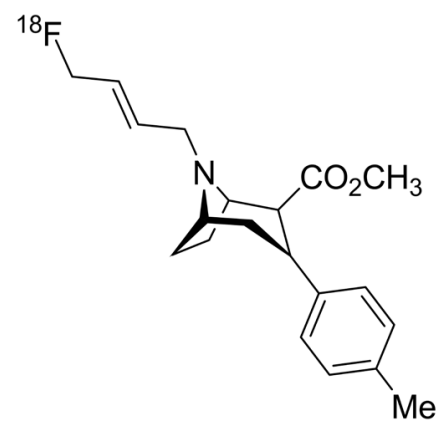

Figure 1. Structure of $\left[{ }^{18} \mathrm{~F}\right] \mathrm{LBT}-999$.

(DAT). It has a great clinical potential in the exploration of dopaminergic neurodegenerative diseases and its metabolism has already been described in rodents [11], non-human primates and ex vivo on human liver microsomes [12].

The aim of this study was the validation of this new approach using UHPLC to quantify interfering radiometabolites in plasma of human subjects, after administration of PET radioligands. First, this manuscript describes the validation process of all the parameters of the new bioanalytical method in order to demonstrate the performance and reliability of its results [13] [14], thus fulfilling ICH requirement about the use of a technique to support the registration of a new drug. Then, we present the preliminary results of $\left[{ }^{18} \mathrm{~F}\right] \mathrm{LBT}-999$ metabolization study in healthy human subjects, carried out concurrently with in vivo PET explorations.

\section{Materials and Methods}

\subsection{Chemicals and Reagents}

Chemicals used for chromatography analysis were certified UHPLC grade. Acetonitrile was purchased from VWR (Radnor, PA, USA), trifluoroacetic acid from Fisher Chemicals (Waltham, MA, USA), and water was obtained by Milli-Q purification (Simplicity SimPack ${ }^{\circledR}$, MerckMillipore, MA, USA). $\left[{ }^{19} \mathrm{~F}\right]$ LBT-999 was prepared and characterized by Villapharma (Spain) and externally qualified by Filab (Dijon, France).

\subsection{Radiolabelling}

Tests and validation were performed with radioactive $\left(\left[{ }^{18} \mathrm{~F}\right] \mathrm{LBT}-999\right)$ and non-radioactive $\left(\left[{ }^{19} \mathrm{~F}\right]\right.$ LBT-999) samples of LBT-999, both formulated in human plasma, and in concentration mimicking in vivo amount in case of patient injection. The radiolabeling process of LBT-999 with fluorine-18 using a TRACERlab FX-FN synthesizer was derived from previously published methods [11] [15], with a mean yield of $35 \%$ (decay corrected) and a mean molar activity of 143.7 $\mathrm{GBq} / \mu \mathrm{mol}$.

\subsection{Online Sample Preparation System}

Plasma samples, obtained by centrifugation of the blood (Heraeus ${ }^{\mathrm{TM}}$ Megafuge $^{\mathrm{TM}}$ 
16R, Thermo Fisher Scientific, Waltham, MA, USA) at $2000 \mathrm{~g}$ for $10 \mathrm{~min}$ at $20^{\circ} \mathrm{C}$, were treated automatically by TFC using an online Turboflow column (Thermo Fisher Scientific, XL C18-P, $1.0 \times 50 \mathrm{~mm}$ column, Waltham, MA, USA) which eliminated the matrix before the injection of the molecule of interest onto the analytical column. Ultrapure water (C) and acetonitrile (D) were used as mobile phase at $4 \mathrm{~mL} / \mathrm{min}$, according to the following gradient: $0-6 \mathrm{~min}$ C:D 100:0 v/v; 6 - 9 min C:D 80:20 v/v; 9 - 15 min C:D 100:0 v/v. During the first $1.5 \mathrm{~min}$, the Turboflow column was loaded with the sample and the matrix eliminated. Then, the valve was switched to transfer the pre-purified sample into the analytical column. During the analysis, the Turboflow column was washed and reconditioned in ultrapure water for the next run.

In order to improve the sensitivity of analysis and to evaluate the extraction efficiency of LBT-999 from plasma by turbulent flow, increasing amounts of samples were injected in duplicate, using one to six sequential loading steps of $100 \mu \mathrm{L}$ each. A solution of $18 \mu \mathrm{g} / \mathrm{mL}$ was used for this study. This concentration is representative of the concentration range determined in vivo by $\mathrm{UV}$ detection at $220 \mathrm{~nm}$. A possible carryover effect was also examined by successively injecting LBT and acetonitrile samples.

\subsection{UHPLC}

Method validation tests and radiometabolites analysis were conducted using a biocompatible UHPLC system Ultimate ${ }^{\mathrm{TM}}$ 3000RS (Thermo Fisher Scientific, Waltham, MA, USA), consisting of one analytical micro pump HPG-3400RS, one loading pump LPG-3400RS, one thermal compartment TCC-3000RS with two switching valves, one autosampler WPS-3000TRS, and two serially connected detectors: one UV/Visible detector VWD-3400RS, equipped with a $11 \mu \mathrm{L}$ flow cell, and one radioactivity detector FlowStar LB513, equipped with a BGO-X $150 \mu \mathrm{L}$ detection cell (Berthold Technologies GmbH \& Co. KG, Thoiry, France). Analysis were carried out with an analytical C18 reverse phase column (Hypersil Gold ${ }^{\circledR} 2.1 \times 100 \mathrm{~mm}, 1.9 \mu \mathrm{m}$, Thermo Fisher Scientific, Waltham, MA, USA), protected by a guard column (Security Guard ${ }^{\mathrm{TM}}$ ULTRA, Phenomenex ${ }^{\circledR}$, Torrance, CA, USA). Trifluoroacetic acid $0.1 \%$ (A) and acetonitrile (B) were used as the mobile phase at $350 \mu \mathrm{L} / \mathrm{min}$, according to the following gradient: 0 $1.5 \min A: B$ 90:10 v/v; 1.5 - 6 min A:B 90:10 $\rightarrow$ 50:50 v/v; 6 - 8 min A:B 50:50 v/v; 8 - 8.3 A:B 50:50 $\rightarrow$ 90:10 v/v; $8.3-15 \mathrm{~min}$ A:B 90:10 v/v. The total run time was 15 minutes. Data acquisition and processing were performed with Chromeleon 7.2 software (Thermo Fisher Scientific, France).

\subsection{Automatic Gamma Counter}

During the study, UHPLC effluent is collected by time at a constant interval of $20 \mathrm{~s}$ and fractions were measured during one minute with an automatic gamma counter (2480 Wizard ${ }^{2}$, Perkin Elmer MA, USA) to confirm the radioactivity detector sensitivity. 


\subsection{Validation Study}

\subsubsection{Extraction Capacity and Matrix Effect}

Turboflow column ability to extract $\left[{ }^{18} \mathrm{~F}\right]$ LBT- 999 from plasma was evaluated by multiple sequential loading. The matrix effect, which is the plasma absorption profile at $220 \mathrm{~nm}$, was also assessed; five filtrated pooled plasma samples were injected in triplicate $(100 \mu \mathrm{L})$ and the mean coefficient of variation of peaks areas was calculated to confirm analysis repeatability. The mean UV absorption of plasma was then subtracted, point by point, from analysis of plasma samples with LBT-999.

\subsubsection{Linearity and Specificity}

Linearity was determined by triplicate analysis of six solutions of LBT-999 in plasma, between 9 and $45 \mu \mathrm{g} / \mathrm{mL}$, representative of the concentration range determined in vivo with UV detection at $220 \mathrm{~nm}$. The calibration curve was constructed to compare injected amount to peak areas.

Specificity was evaluated through the retention time (RT) of LBT-999 at 220 nm.

\subsubsection{Precision}

The precision of the method was assessed by intra-day precision or repeatability, and intermediate precision. The repeatability is defined as the closeness of agreement of peak area between 3 series of analysis in the same operating conditions over a short interval of time. The intermediate precision defines the same variation for two experiments carried out on two different days. Both are expressed as coefficients of variation $\left(\mathrm{CV}(\%)=\frac{\text { Standard deviation }}{\text { Mean }}\right)$.

\subsubsection{Sensitivity}

Sensitivity of the online radioactivity detector was determined by analyzing samples of calibrated activities. The gamma-counter was used to precisely calibrate samples' activity. The value of the signal recorded by the online radiodetector (in cps) was then correlated to the activity of the analyzed sample. Two limits were set according to International Conference on Harmonization (ICH) guidelines [16]: the limit of detection (LOD) and the Lower limit of quantification (LLOQ), respectively defined as 3 and 10 times the background noise (or signal to noise ratio $\mathrm{S} / \mathrm{N}=3$ and $\mathrm{S} / \mathrm{N}=10$ ).

\subsubsection{Statistics}

Descriptive statistical analysis was performed using Prism software (GraphPad, CA USA). Data from linearity were evaluated by calculating the correlation coefficient $\left(\mathrm{r}^{2}\right)$ of the calibration curve by linear regression. A p-value $<0.05$ was indicative of a statistically significant correlation. The CV of the identification parameters for LBT-999, RT, was considered acceptable if around 1\%. In the context of the first validation steps of a bioanalytical method, higher values of $\mathrm{CV}$ were considered acceptable for quantitative parameters like amount precision [16]. 


\subsection{Clinical Study}

\subsubsection{Human Subjects}

Four healthy volunteers ( 2 men, 2 women; age, 47 - 79 y; weight, $83.5 \pm 12 \mathrm{~kg}$ ) free of any neuropsychiatric or other medical condition, provided written informed consent to participate to a monocentric study approved by the French National Agency for Medicines Safety (ref. number 141363A-12) and the local Ethics Committee (ref. number 2014-R29). All were subjected to a physical examination and structural brain MRI.

\subsubsection{Radiometabolite Analysis}

Concomitantly to PET acquisition, $5 \mathrm{~mL}$ samples of venous blood were manually drawn through a venous cannula in the opposite arm of injection, at 5, 15, 30 and $45 \mathrm{~min}$ for radiometabolites evaluation. With the method described earlier, $300 \mu \mathrm{L}$ of plasma from each time-point were injected in the system for analysis. UHPLC effluent is collected by time at a constant interval of $20 \mathrm{~s}$ for parallel gamma-counter measurements. Proportion of unchanged $\left[{ }^{18} \mathrm{~F}\right] \mathrm{LBT}-999$ and new formed radiometabolites were calculated as fraction of total areas of all detected peaks, based on the UHPLC radiochromatograms and gamma-counter results.

\section{Results}

\subsection{Validation}

\subsubsection{Turboflow Column Extraction Capacity and Matrix Effect}

Testing of the Turboflow column showed no carryover between the successive analysis of LBT-999 and acetonitrile. Losses of LBT-999, extrapolated from peak area, after multiple sequential loading on the Turboflow column were resumed in Table 1. Average amount of LBT-999 extracted by the Turboflow column was $80 \% \pm 9 \%$.

During the evaluation of the matrix effect, CV of peak areas of UHPLC-UV analysis of plasma samples were below $1 \%$, Figure 2 presents the matrix corrected UHPLC-UV profile of LBT-999 in plasma.

\subsubsection{Linearity and Specificity}

Several experiments with three sequential loading of $100 \mu \mathrm{L}$ were performed to evaluate UV and radio-detection linearity; respectively three experiments with $\left[{ }^{19} \mathrm{~F}\right] \mathrm{LBT}-999$ between 9 and $45 \mu \mathrm{g} / \mathrm{mL}$, and one experiment with $\left[{ }^{18} \mathrm{~F}\right] \mathrm{LBT}-999$

Table 1. Effect of multiple sequential loading on the Turboflow column.

\begin{tabular}{cc}
\hline Number of loads & Extracted LBT-999 (\%) \\
\hline 1 & 100 \\
2 & 97 \\
3 & 88 \\
4 & 75 \\
6 & 41 \\
\hline
\end{tabular}


between 2.4 and $8.6 \mathrm{kBq} / \mathrm{mL}$. Linearity of LBT-999 and $\left[{ }^{18} \mathrm{~F}\right] \mathrm{LBT}-999$ mean peak area are represented respectively on Figure 3 and Figure 4 . Coefficients of correlation $\mathrm{r}^{2}$ were respectively 0.997 and 0.972 , with a $\mathrm{p}$-value $<0.01$.

\subsubsection{Precision}

CV expressing the repeatability of LBT-999 peak areas in UV detection were inferior to $4 \%$. The intermediate precision, evaluated by three similar experiments on three different days, led to a mean CV of $30.9 \%$ for each standard.

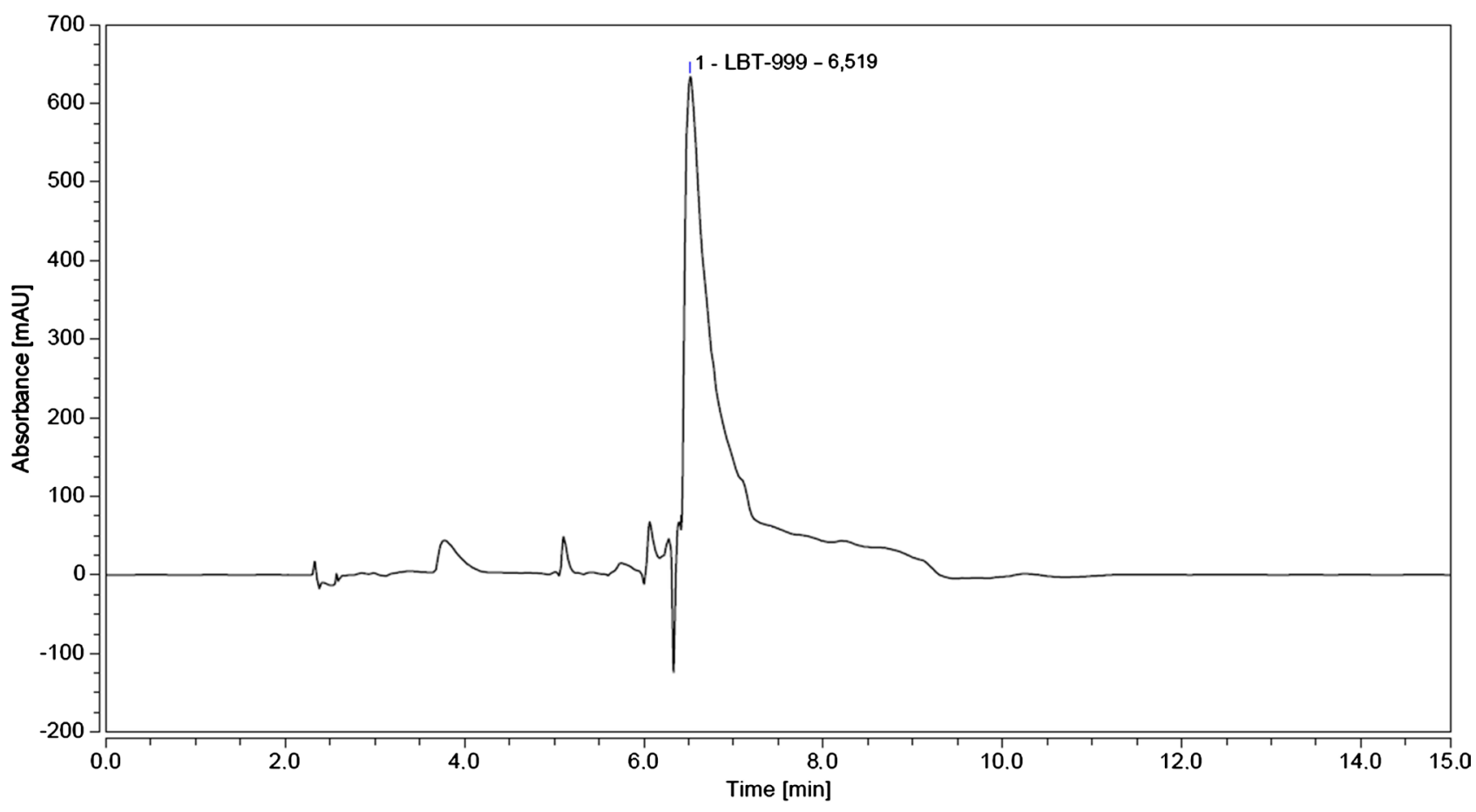

Figure 2. UHPLC-UV (220 nm) of LBT-999 with plasma matrix correction.

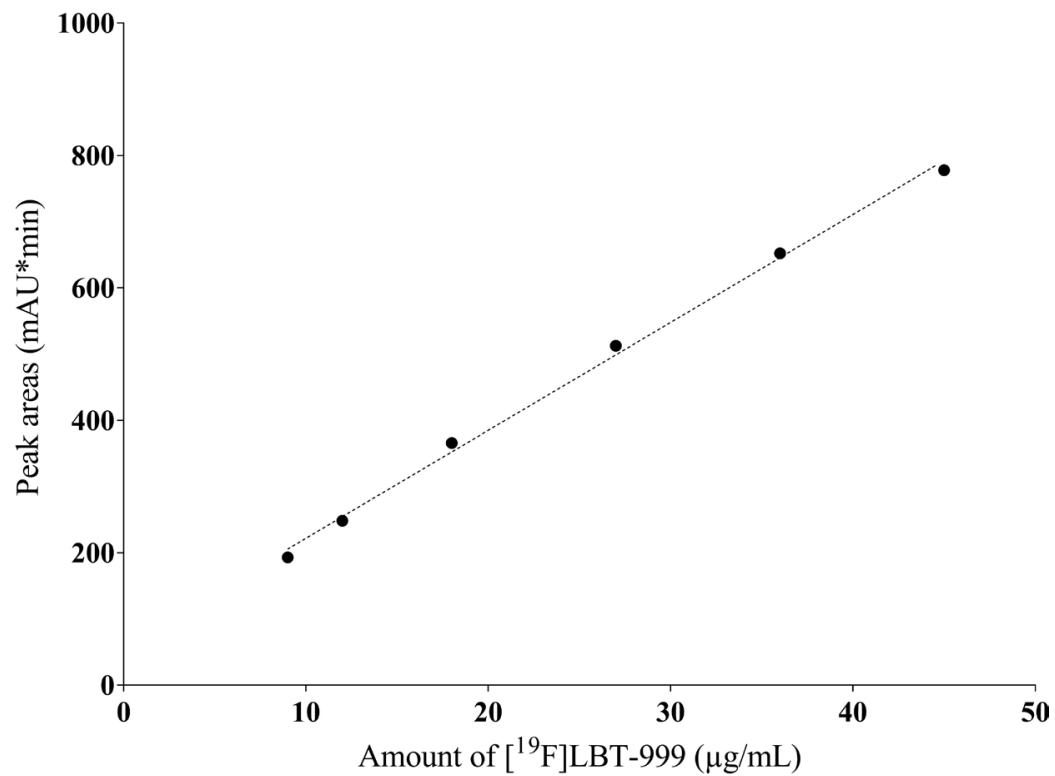

Figure 3. Linear regression of peak areas for $\left[{ }^{19} \mathrm{~F}\right]$ LBT-999 by UV detection. 


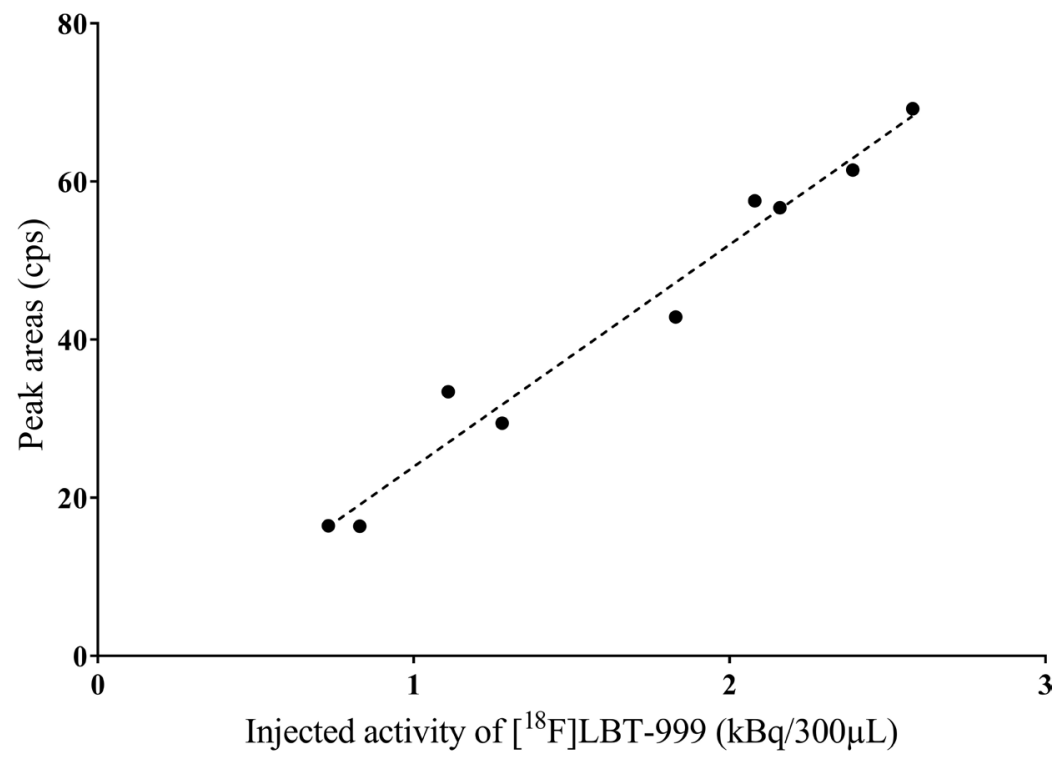

Figure 4. Linear regressionof peak areas for $\left[{ }^{18} \mathrm{~F}\right] \mathrm{LBT}-999$ by radio-detection.

\subsubsection{Sensitivity}

The radiodetector sensitivity was established on the average noise level of $3 \mathrm{cps}$, setting LOD and LLOQ at $9 \mathrm{cps}$ and $30 \mathrm{cps}$. Plasma samples were calibrated with the gamma-counter and analyzed in radio-UHPLC to determine the radiodetector limits. Mean background noise was graphically evaluated at $3 \mathrm{cps}$, and LOD and LLOQ were thus set at $0.3 \mathrm{kBq}$ and $1 \mathrm{kBq}$, respectively.

\subsection{Clinical Study}

\section{$\left[{ }^{18}\right.$ F] LBT-999 Radiometabolites analysis}

Radio-UHPLC analysis of plasma from the 4 subjects injected with $\left[{ }^{18} \mathrm{~F}\right] \mathrm{LBT}-999$ showed two minor radiometabolite peaks along with $\left[{ }^{18} \mathrm{~F}\right] \mathrm{LBT}-999$ (mean RT 6.5 $\pm 0.1 \mathrm{~min})$. The main radiometabolite was more hydrophilic than $\left[{ }^{18} \mathrm{~F}\right] \mathrm{LBT}-999$, with a mean RT of $4.8 \mathrm{~min}$, and the other one was more lipophilic (mean RT 9.6 min) (Figure 5). The plasmatic fraction of intact parent molecule decreases regularly but slowly over time, from $100 \%$ at 5 min post-injection (pi) to $57.8 \% \pm$ $25 \%$ at $45 \mathrm{~min}$ pi; so it does remained predominant for most of the exam duration. In addition to the two above-mentioned radiometabolites revealed by radio-UHPLC, the formation of another polar radiometabolite was suspected. It was eluted at $2.2 \mathrm{~min}$, appeared $30 \mathrm{~min}$ after the injection of $\left[{ }^{18} \mathrm{~F}\right] \mathrm{LBT}-999$ and represented 5 to $11 \%$ of the global radioactivity. Although peaks were hardly emerging from the noise baseline, RT were repeatable and gamma-counter measurement of the fractionated eluate after radio-UHPLC separation was used as a gold standard to confirm the radioactivity detector results.

\section{Discussion}

This work was designed firstly for validation of a new bioanalytical method evaluating drug metabolism in human blood using UHPLC with prior sample preparation 


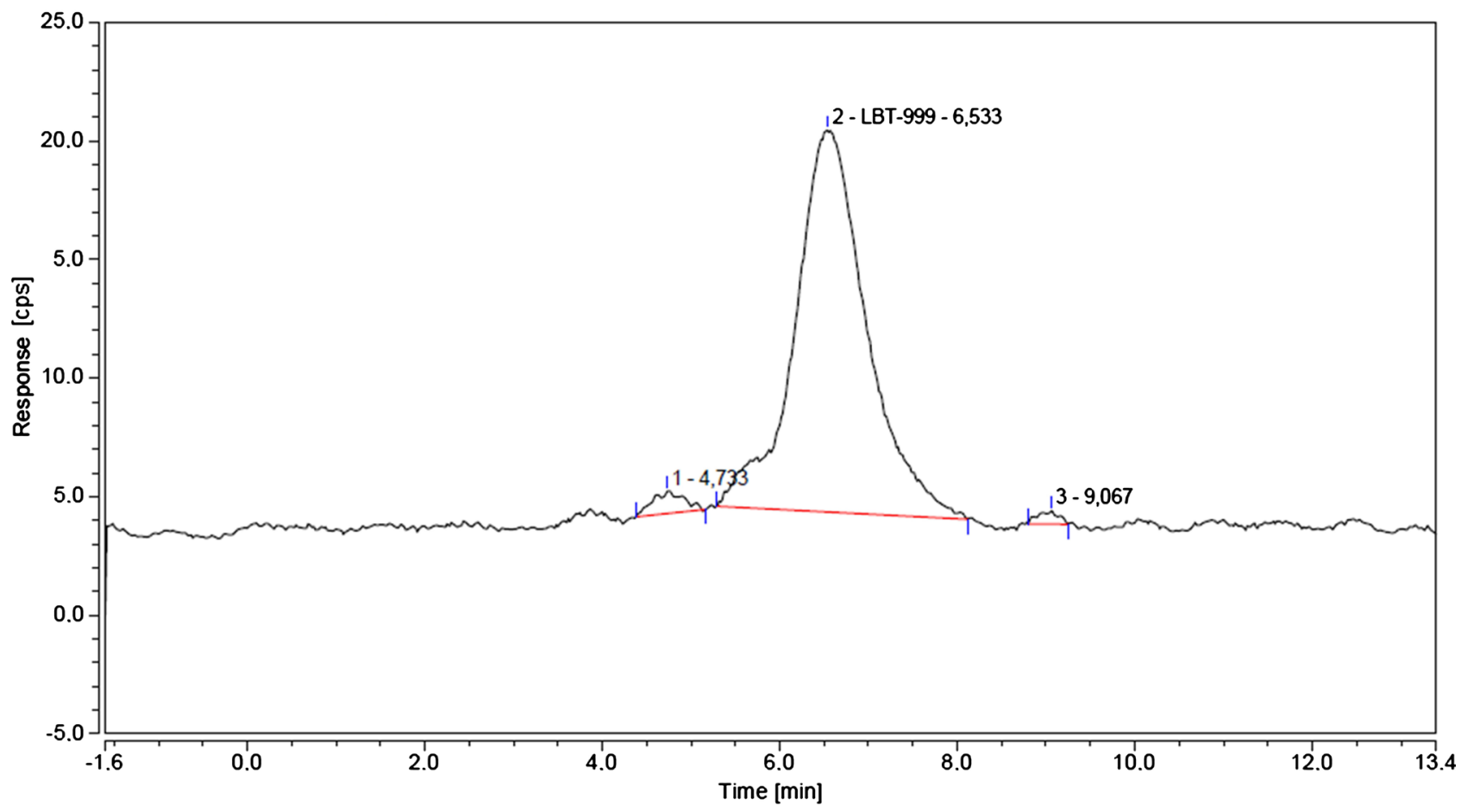

Figure 5. Radio-UHPLC of a sample of blood withdrawn 15 minutes after the injection of $\left[{ }^{18} \mathrm{~F}\right] \mathrm{LBT}-999$; we observed the major peak of $\left[{ }^{18} \mathrm{~F}\right] \mathrm{LBT}-999$ at $6.5 \mathrm{~min}$, and 2 minor peaks of radiometabolites at 4.7 and $9.1 \mathrm{~min}$.

limited to online deproteinization by turbulent flow clean-up, and secondly for the preliminary study of $\left[{ }^{18} \mathrm{~F}\right] \mathrm{LBT}-999$ metabolism in human subjects. Lipophilic radiometabolites, after crossing the $\mathrm{BBB}$, may interfere with the specific brain PET signal or increase the non-specific uptake, then altering both quantification and signal to noise ratio $(\mathrm{S} / \mathrm{N})$. Hence, challenging PET images interpretation and diagnosis accuracy. Thus, the development and implementation of a robust analytical method to evaluate metabolism and accurately quantify PET signal is a crucial step in the in vivo evaluation of a new brain PET radioligand. This process entails the determination and validation of several parameters conditioning its liability and robustness.

Compared to conventional HPLC, UHPLC analysis presents several advantages. Indeed, UHPLC columns have smaller particles ( $<2 \mu \mathrm{m}$ vs. $>5 \mu \mathrm{m}$ ), which compels to work with higher pressure, but induces sharper peaks. Thus, analysis resolution is enhanced, resulting potentially in a more accurate metabolites separation. Solvent consumption is also reduced thanks to lower diameter and length of the column $(0.350 \mathrm{~mL} / \mathrm{min}$ vs. $1 \mathrm{~mL} / \mathrm{min})$ and shorter run time. To optimize compounds separation, a gradient of mobile phase was implemented. Column and mobile phase were carefully chosen to avoid retention or dispersion in the dead volume of substances, e.g. free fluorine originating from defluorination, and allow exhaustive analysis.

The evaluation and minimization of matrix effects, mostly due to endogenous phospholipids [17], is another crucial challenge in quantitative analysis.

For each experiment, matrix absorption at $220 \mathrm{~nm}$ (UHPLC-UV) was deter- 
mined and subtracted from every sample analysis. An online preparation system ensures a better repeatability, reduces the loss of information in samples analysis, and offers better radiation protection by limiting manual intervention. Usually, biological sample treatment procedures are protein precipitation (PPT), liquid-liquid extraction (LLE) and solid phase extraction (SPE). Of these three approaches, SPE is the one presenting the better selectivity due to its versatility. Automated online SPE is often used for high throughput analysis, but sorbents are not usually well adapted to deal with biological samples. Therefore in the mid 1990's, TFC Turboflow columns, filled with large particles of stationary phase, were developed. They allow the use of high flow rate, lowering the molecular weight exclusion cut-off during the preparation without high back pressure. A turbulent flow is generated, which induces a fast transfer of small molecules of interest into the pores of the stationary phase where they are retained depending on their affinity for the bonded phase. Due to their low molecular diffusion coefficient, larger molecules like phospholipids or proteins don't diffuse in these pores and are rapidly eliminated by the turbulent flow [17]. Depending on the loading flow rate and $\mathrm{pH}$, more than $94 \%$ and $99 \%$ of proteins with a molecular weight greater than 1 and $15 \mathrm{kDa}$ respectively, are not retained by the Turboflow column [18]. Thus, only the molecules of interest are transferred in the UHPLC and analyzed. As it has been demonstrated several times [19] [20], turbulent flow shows a better efficiency compared to LLE or SPE for the effective removal of plasmatic proteins in samples, is reproducible and allows fast sample extraction process, which deals better with the complexity of biological samples. It also confers several other advantages. Firstly, fast analysis process are of crucial consideration when working with short half-time isotopes. Secondly, the significantly reduced amount of plasmatic protein injected into the system preserves it to a large extend from built-up pressure or clogging due to proteins deposits. Moreover, only the unconjugated drug is extracted and then analyzed using the turbulent flow technology. This free drug fraction is also the portion that crosses the BBB, which is the source of brain PET signal. Thus, it was of interest to conduct our experimentations with a radiopharmaceutical for neuroimaging applications.

Along with the Turboflow column, another technique of sample preparation by PPT with zinc sulfate $\left(\mathrm{ZnSO}_{4}\right)$ and methanol has still been considered to optimize proteins removal [21], drug extraction efficiency from plasma, detection sensitivity [22] and assay robustness [23]. With this non-selective pretreatment, recovered plasma was colorless and translucent; red blood cells were lysed and plasmatic proteins fully precipitated [24] [25]. Nevertheless, this additional manual step lengthens the preparation process and may lower the sample throughput. In-house in vitro assays showed a mean recovery of $82 \%$ of $\left[{ }^{18} \mathrm{~F}\right] \mathrm{FDG}$ from plasma (data not shown). As an alternative to the turbulent flow system, microSPE can also be used to analyze metabolites in blood samples. But, even with this method, a sample pretreatment including plasmatic protein precipitation is re- 
quired [26]. For radiometabolites analysis, other authors [27] used fast-LC which shortened greatly the run time but still required a step of sample deproteinization that can increase preparation variability. Direct plasma injection combined with micellar cleanup in fast-LC [28] also showed promises, but was sparsely described in literature and more studies may be required to validate this approach.

A good detection sensitivity is essential for an accurate evaluation of radiometabolites; indeed, measured signals may be weak for several reasons. Firstly, the fast radioactive decay of the isotope, which calls for quick sampling, sample preparation and analysis. Secondly, experiments may be one or two hours long and require the analysis of several biological samples from later time points where even minor metabolites should be detected. And thirdly, the sample volume activity (VA), directly influenced by the blood volume of the studied species. Thus, in preclinical metabolism studies, high VA are observed, and challenges arise only with small animals when the total amount of blood drawn is very low. But in humans, expected VA values are much lower. Therefore, sensitivity of detection becomes a key parameter in radiometabolites evaluation and the analytical process development. Sensitivity could be enhanced either by using a larger detection cell or by concentrating sample. The first approach leads to widening peaks on chromatograms, thus decreasing resolution and detectability. Sample concentration, by multiple sequential loads onto the Turboflow column prior to transfer on the analytical column, was then privileged. The number of consecutive loading steps was limited to three; beyond three, more than $15 \%$ of LBT-999 was not retained on the Turboflow column. Moreover, with each loading analysis duration lengthens and risk of system clogging or sample breakthrough increases.

We demonstrated that the radioactivity detector' threshold in these work conditions was $1 \mathrm{kBq}$ for quantification, which presents adequate sensitivity to analyze samples with the smallest activity measured during experiments $(3 \mathrm{kBq} / 300 \mu \mathrm{L})$. However, additional experiments are required to improve sensitivity for low VA samples, for example in case of minor metabolites or later time points.

The method linearity was confirmed; the coefficient of correlation $\left(\mathrm{r}^{2}\right)$ obtained by linear regression was high with a p-value $<0.05$. Because of low plasma availability, solutions had to be redone for each experimentation, possibly increasing the variation of their concentrations. The method specificity was verified; variation of LBT-999 RT were not significant nor in UV $\left(\mathrm{CV}_{\mathrm{UV}}=1.2 \%\right)$ nor in radio $\left(\mathrm{CV}_{\text {Radio }}=0.5 \%\right)$. Although tests showed a high repeatability, inter-day precision presented a high CV of $30.9 \%$. An analytical technique with good precision provides comparable results for the analysis of similar samples. Poor intermediate precision of a test may wrongly generate significant variation between two experimentations, entailing inaccurate conclusions. However, fast radioactive decay of fluorine-18 and low plasma supplies usually prevented us from duplicating tests on a single time point sampling. With the high repeatabil- 
ity of the method, it is possible to conduct experiments within a day, for example the study of preclinical metabolism. The lack of inter-day precision has a stronger impact on the evaluation of in-human metabolism, for which only samples from one single patient are injected within a day. The number of experiments in this study was limited; additional analysis should be performed to reduce the $\mathrm{CV}$ between peak areas, improve the method precision and validate the method accuracy. As part of the first validation of an innovative bioanalytical method to quantify radiometabolites in human slightly superior CV are acceptable.

After the qualification process, this bioanalytical method was tested for the evaluation of $\left[{ }^{18} \mathrm{~F}\right]$ LBT-999 in-human metabolism. Thanks to radio-UHPLC analysis and gamma counter measures, three radiometabolites were detected, two of which were more polar than $\left[{ }^{18} \mathrm{~F}\right] \mathrm{LBT}-999$. The parent fraction represented $60 \%$ of the total radioactivity at 30 minutes pi. Overall, the metabolism appears favorable to clinical imaging. These findings, although preliminary, are congruent with studies on in vitro and preclinical metabolism of $\left[{ }^{18} \mathrm{~F}\right] \mathrm{LBT}-999$ [12] [29]. They are also consistent with in-human metabolism of radiopharmaceuticals belonging to the same chemical class, $\left[{ }^{11} \mathrm{C}\right]$ PE2I and $\left[{ }^{18} \mathrm{~F}\right] \mathrm{FE}-\mathrm{PE} 2 \mathrm{I}$, targeting DAT [30].

Indeed, in vitro $\left[{ }^{18} \mathrm{~F}\right] \mathrm{LBT}-999$ study with liver microsomes and its preclinical characterization in rodents and baboons showed 5 polar metabolites including 4 and 3 radiometabolites, respectively. Two major transformations were identified: hydroxylation of both tolyl group and tropane ring by isoform CYP2D6 and $N$-dealkylation of the tropane ring by CYP3A4 [29] [31]. They occurred in peripheral blood and then metabolites crossed the BBB. Thirty minutes after the injection, around $50 \%$ of parent $\left[{ }^{18} \mathrm{~F}\right] \mathrm{LBT}-999$ was already transformed [12]; metabolism level was considered significant, and started shortly after the injection of the drug.

Injections of $\left[{ }^{11} \mathrm{C}\right] \mathrm{PE} 2 \mathrm{I}$ in animals and humans led to the formation of five polar metabolites, out of which four are radiolabeled and only two were found in the striatum [32]. The first radiometabolite was created by the hydroxylation of $\left[{ }^{11} \mathrm{C}\right] \mathrm{PE} 2 \mathrm{I}$ by liver CyP450, forming a 4-hydroxymethyl analog presenting an intermediate lipophilicity $(\log \mathrm{D}=2.7)$ and so being able to cross the $\mathrm{BBB}$. The prominent accumulation of this radiometabolite in the striatum indicates its high affinity for DAT. The second radiometabolite was a 4-carboxy-PE2I analog resulting from the action of alcohol and aldehyde dehydrogenases in the brain on the first radiometabolite. The 4-carboxy analog was responsible for non-specific uptake in the cerebellum. The three other metabolites, resulting from $N$-dealkylation and esterification, were only found in plasma and urine. The in vivo metabolism rate of $\left[{ }^{11} \mathrm{C}\right]$ PE2I appears faster than $\left[{ }^{18} \mathrm{~F}\right] \mathrm{LBT}-999$, since $65 \%$ of the parent molecule are already transformed 10 minutes after injection [33] [34].

$\left[{ }^{18} \mathrm{~F}\right] \mathrm{FE}-\mathrm{PE} 2 \mathrm{I}$, synthesized after chemical modulation of the tropane ring, presented a metabolism in humans similar to $\left[{ }^{11} \mathrm{C}\right] \mathrm{PE} 2 \mathrm{I}^{\prime} \mathrm{s}$. The same enzymes, liver CyP450 and cerebral dehydrogenases, respectively produced 4-hydroxymethyl and 4-carboxy analogs of $\left[{ }^{18} \mathrm{~F}\right] \mathrm{FE}-\mathrm{PE} 2 \mathrm{I}$, the two radiometabolites identified in 
the brain [5] [35]. Thirty minutes after the injection, parent $\left[{ }^{18} \mathrm{~F}\right] \mathrm{FE}-\mathrm{PE} 2 \mathrm{I}$ represented only $14 \%$ of the radioactivity [5]. In vitro and preclinical tests allowed to detect the formation of at least three other minor radiometabolites, originating mainly from $N$-dealkylation and benzyl hydroxylation [36] [37].

This brief review of literature corroborate our results and hereby ascertain the robustness of our method. As expected, metabolism of $\left[{ }^{18} \mathrm{~F}\right] \mathrm{LBT}-999$ is more extensive in preclinical and in vitro evaluation than in human organism [38]. But $\left[{ }^{18} \mathrm{~F}\right]$ LBT-999 also appears more stable with a slower transformation rate, which can allow the reduction of the injected dose and a better image resolution. The next step is now to identify all metabolites, usually by mass spectrometry, to confirm involved enzymes.

\section{Conclusion}

We developed a new method to quantify radiometabolites over time using UHPLC and turbulent flow technology for online sample preparation, instead of conventional HPLC and manual sample preparation. High extraction efficiency of the drug from the plasma and multiple loading in the Turboflow column allowed the analysis of sample of low concentrations thanks to a concentration process. Analytical conditions were highly repeatable, with invariable retention time and a linear calibration proving the method specificity and proportionality to peak areas, respectively. Additional tests are necessary to validate intermediate precision and maybe enhance detection sensitivity. This innovative technique of sample preparation and analysis opens new perspectives for radiometabolites quantification. Finally, although results of the first clinical trial are preliminary and need to be confirmed, metabolism of $\left[{ }^{18} \mathrm{~F}\right] \mathrm{LBT}-999$ is in line with expectations and might even exceed them; $\left[{ }^{18} \mathrm{~F}\right]$ LBT-999 kinetics appears favorable to neuroimaging and its metabolism slow and less extensive than previous radiotracers.

\section{Acknowledgements}

This study was supported by the French National Agency for Research ("Investissement d'Avenir" no. ANR-11-LABX-0018-01), IRON.

\section{Funding}

This study was supported by the French National Agency for Research (INSERM) CVT Aviesan/DVS Agents d'imagerie.

\section{Conflicts of Interest}

Claude Netter holds a position as an employee in Thermo Fisher Scientific Company. The other authors declare no potential conflicts of interest.

\section{References}

[1] Mankoff, D.A. (2007) A Definition of Molecular Imaging. Journal of Nuclear Medi- 
cine, $48,18 \mathrm{~N}-21 \mathrm{~N}$.

[2] Wu, C., Li, F., Niu, G. and Chen, X. (2013) PET Imaging of Inflammation Biomarkers. Theranostics, 3, 448-466. https://doi.org/10.7150/thno.6592

[3] Meadows, N., Morrison, A., Brindley, D., Schuh, A. and Barker, R. (2015) An Evaluation of Regulatory and Commercial Barriers to Stratified Medicine Development and Adoption. The Pharmacogenomics Journal, 15, 6-12. https://doi.org/10.1038/tpj.2014.51

[4] Bertrán, M., Martínez, N., Carbajal, G., Fernández, A. and Gómez, Á. (2016) An Open Tool for Input Function Estimation and Quantification of Dynamic PET FDG Brain Scans. International Journal of Computer Assisted Radiology and Surgery, 11, 1419-1430. https://doi.org/10.1007/s11548-015-1307-x

[5] Sasaki, T., Ito, H., Kimura, Y., Arakawa, R., Takano, H., Seki, C., et al. (2012) Quantification of Dopamine Transporter in Human Brain Using PET with ${ }^{18} \mathrm{~F}-\mathrm{FE}-\mathrm{PE} 2 \mathrm{I}$. Journal of Nuclear Medicine, 53, 1065-1073. https://doi.org/10.2967/jnumed.111.101626

[6] Pike, V.W. (2009) PET Radiotracers: Crossing the Blood-Brain Barrier and Surviving Metabolism. Trends in Pharmacological Sciences, 30, 431-440. https://doi.org/10.1016/j.tips.2009.05.005

[7] Lemke, T.L. and Williams, D.A. (2008) Drug Metabolism. In: Foye's Principles of Medicinal Chemistry. Lippincott Williams \& Wilkins, Philadelphia, PA.

[8] Clarke, D. and Sokoloff, L. (1999) Substrates of Cerebral Metabolism. In: Siegel, G.J., Agranoff, B.W., Albers, R.W., Fisher, S.K. and Uhler, M.D., Eds., Basic Neurochemistry: Molecular, Cellular and Medical Aspects, 6th Edition, Lippincott-Raven, Philadelphia, PA. https://www.ncbi.nlm.nih.gov/books/NBK28048/

[9] Rokka, J., Grönroos, T.J., Viljanen, T., Solin, O. and Haaparanta-Solin, M. (2017) HPLC and TLC Methods for Analysis of $\left[{ }^{18} \mathrm{~F}\right] \mathrm{FDG}$ and Its Metabolites from Biological Samples. Journal of Chromatography B, 1048, 140-149.

https://doi.org/10.1016/j.jchromb.2017.01.042

[10] Collste, K., Forsberg, A., Varrone, A., Amini, N., Aeinehband, S., Yakushev, I., Halldin, C., Farde, L. and Cervenka, S. (2016) Test-Retest Reproducibility of $\left[{ }^{11} \mathrm{C}\right]$ PBR28 Binding to TSPO in Healthy Control Subjects. European Journal of Nuclear Medicine and Molecular Imaging, 43, 173-183. https://doi.org/10.1007/s00259-015-3149-8

[11] Sérrière, S., Tauber, C., Vercouillie, J., Guilloteau, D., Deloye, J.-B., Garreau, L. and Chalon, S. (2014) In Vivo PET Quantification of the Dopamine Transporter in Rat Brain with $\left[{ }^{18} \mathrm{~F}\right] \mathrm{LBT}-999$. Nuclear Medicine and Biology, 41, 106-113. https://doi.org/10.1016/j.nucmedbio.2013.09.007

[12] Peyronneau, M-A., Saba, W., Dollé, F., Goutal, S., Coulon, C., Bottlaender, M. and Valette, H. (2012) Difficulties in DAT Radioligand PET Analysis: The Example of LBT-999 Using $\left[{ }^{18} \mathrm{~F}\right]$ and $\left[{ }^{11} \mathrm{C}\right]$ Labelling-Part II. Nuclear Medicine and Biology, 39, 347-359. https://doi.org/10.1016/j.nucmedbio.2011.09.006

[13] Sonawane, L.V., Poul, B.N., Usnale, S.V., Waghmare, P.V. and Surwase, L.H. (2014) Bioanalytical Method Validation and Its Pharmaceutical Application-A Review. Pharmaceutica Analytica Acta, 5. https://doi.org/10.4172/2153-2435.1000288 https://www.omicsonline.org/open-access/bioanalytical-method-validation-and-itspharmaceutical-application-a-review-2153-2435.1000288.php?aid=25188

[14] Shah, V.P. (2007) The History of Bioanalytical Method Validation and Regulation: Evolution of a Guidance Document on Bioanalytical Methods Validation. The AAPS Journal, 9, E43-E47. https://doi.org/10.1208/aapsj0901005 
[15] Varrone, A., Stepanov, V., Nakao, R., Toth, M., Gulyas, B., Emond, P., et al. (2011) Imaging of the Striatal and Extrastriatal Dopamine Transporter with ${ }^{18} \mathrm{~F}-\mathrm{LBT}-999$ : Quantification, Biodistribution, and Radiation Dosimetry in Nonhuman Primates. Journal of Nuclear Medicine, 52, 1313-1321. https://doi.org/10.2967/jnumed.111.089953

[16] ICH Harmonized Tripartite (2005) Validation of Analytical Procedures: Text and Methodology Q2 R1.

https://www.ich.org/fileadmin/Public_Web_Site/ICH_Products/Guidelines/Quality /Q2_R1/Step4/Q2_R1__Guideline.pdf

[17] Couchman, L. (2012) Turbulent Flow Chromatography in Bioanalysis: A Review. Biomedical Chromatography, 26, 892-905. https://doi.org/10.1002/bmc.2769

[18] Herman, J., Edge, T. and Majors, R. (2012) Theoretical Concepts and Applications of Turbulent Flow Chromatography. Lc Gc North America, 3, 200-214.

[19] Michopoulos, F., Edge, A.M., Theodoridis, G. and Wilson, I.D. (2010) Application of Turbulent Flow Chromatography to the Metabonomic Analysis of Human Plasma: Comparison with Protein Precipitation. Journal of Separation Science, 33, 1472-1479. https://doi.org/10.1002/jssc.200900789

[20] Liesener, A. and Karst, U. (2005) Turbulent Flow Chromatography for the Reduction of Matrix Effects in Electrospray Ionization Mass Spectrometry-Based Enzyme Assays. Journal of Separation Science, 28, 1658-1665. https://doi.org/10.1002/jssc.200500090

[21] Du, L. and White, R.L. (2008) Reducing Glycerophosphocholine Lipid Matrix Interference Effects in Biological Fluid Assays by Using High-Turbulence Liquid Chromatography. Rapid Communications in Mass Spectrometry, 22, 3362-3370. https://doi.org/10.1002/rcm.3747

[22] He, X. and Kozak, M. (2012) Development of a Liquid Chromatography Tandem Mass Spectrometry Method for Plasma-Free Metanephrines with Ion-Pairing Turbulent Flow Online Extraction. Analytical and Bioanalytical Chemistry, 402, 3003-3010. https://doi.org/10.1007/s00216-012-5768-2

[23] Bourgogne, E., Soichot, M., Latour, C. and Laprévote, O. (2013) Rugged and Accurate Quantitation of Colchicine in Human Plasma to Support Colchicine Poisoning Monitoring by Using Turbulent-Flow LC-MS/MS Analysis. Bioanalysis, 5, 2889-2896. https://doi.org/10.4155/bio.13.258

[24] Polson, C., Sarkar, P., Incledon, B., Raguvaran, V. and Grant, R. (2003) Optimization of Protein Precipitation Based upon Effectiveness of Protein Removal and Ionization Effect in Liquid Chromatography-Tandem Mass Spectrometry. Journal of Chromatography B, 785, 263-275. https://doi.org/10.1016/S1570-0232(02)00914-5

[25] Annesley, T.M. and Clayton, L. (2004) Simple Extraction Protocol for Analysis of Immunosuppressant Drugs in Whole Blood. Clinical Chemistry, 50, 1845-1848. https://doi.org/10.1373/clinchem.2004.037416

[26] Xie, X., Carrell, T. and Kozak, M. (2016) Quantitation of THC and THC Metabolites in Blood Using SOLA $\mu$ SPE Plates and the TSQ Quantiva Triple Quadrupole Mass Spectrometer for Forensic Analysis. Thermo Fisher Scientific, 657, 1-6.

[27] Nakao, R., Schou, M. and Halldin, C. (2012) Rapid and Sensitive Measurement of PET Radioligands in Plasma by Fast Liquid Chromatography/Radiometric Detection. Journal of Chromatography B, 895-896, 116-122. https://doi.org/10.1016/j.jchromb.2012.03.028

[28] Nakao, R., Schou, M. and Halldin, C. (2012) Rapid Metabolite Analysis of Positron 
Emission Tomography Radioligands by Direct Plasma Injection Combining Micellar Cleanup with High Submicellar Liquid Chromatography with Radiometric Detection. Journal of Chromatography A, 1266, 76-83. https://doi.org/10.1016/j.chroma.2012.10.022

[29] Saba, W., Peyronneau, M.-A., Dollé, F., Goutal, S., Bottlaender, M. and Valette, H. (2012) Difficulties in DAT Radioligand PET Analysis: LBT-999 Using $\left[{ }^{18} \mathrm{~F}\right]$ and $\left[{ }^{11} \mathrm{C}\right]$ Labelling-Part I. Nuclear Medicine and Biology, 39, 227-233. https://doi.org/10.1016/j.nucmedbio.2011.08.003

[30] Schou, M., Steiger, C., Varrone, A., Guilloteau, D. and Halldin, C. (2009) Synthesis, Radiolabeling and Preliminary in Vivo Evaluation of $\left[{ }^{18} \mathrm{~F}\right] \mathrm{FE}-\mathrm{PE} 2 \mathrm{I}$, a New Probe for the Dopamine Transporter. Bioorganic \& Medicinal Chemistry Letters, 19, 4843-4845. https://doi.org/10.1016/j.bmcl.2009.06.032

[31] Zoghbi, S.S., Shetty, H., Ichise, M., Fujita, M., Imaizumi, M., Liow, J.S., et al. (2006) PET Imaging of the DAT with ${ }^{18}$ F-FECNT: A Polar Radiometabolite Confounds Brain Radioligand Measurements. Journal of Nuclear Medicine, 47, 520-527.

[32] Shetty, H.U., Zoghbi, S.S., Liow, J.-S., Ichise, M., Hong, J., Musachio, J.L., et al. (2007) Identification and Regional Distribution in Rat Brain of Radiometabolites of the Dopamine Transporter PET Radioligand $\left[{ }^{11} \mathrm{C}\right]$ PE2I. European Journal of Nuclear Medicine and Molecular Imaging, 34, 667-678. https://doi.org/10.1007/s00259-006-0277-1

[33] Hirvonen, J., Johansson, J., Teräs, M., Oikonen, V., Lumme, V., Virsu, P., et al. (2008) Measurement of Striatal and Extrastriatal Dopamine Transporter Binding with High-Resolution PET and $\left[{ }^{11} \mathrm{C}\right]$ PE2I: Quantitative Modeling and Test-Retest Reproducibility. Journal of Cerebral Blood Flow \& Metabolism, 28, 1059-1069. https://doi.org/10.1038/sj.jcbfm.9600607

[34] Jucaite, A., Odano, I., Olsson, H., Pauli, S., Halldin, C. and Farde, L. (2006) Quantitative Analyses of Regional $\left[{ }^{11} \mathrm{C}\right] \mathrm{PE} 2 \mathrm{I}$ Binding to the Dopamine Transporter in the Human Brain: A PET Study. European Journal of Nuclear Medicine and Molecular Imaging, 33, 657-668. https://doi.org/10.1007/s00259-005-0027-9

[35] Fazio, P., Svenningsson, P., Forsberg, A., Jonsson, E.G., Amini, N., Nakao, R., et al. (2015) Quantitative Analysis of ${ }^{18} \mathrm{~F}-(E)-N$-(3-Iodoprop-2-Enyl)-2 $\beta$-Carbofluoroethoxy-3 $\beta$-(4'-Methyl-Phenyl) Nortropane Binding to the Dopamine Transporter in Parkinson Disease. Journal of Nuclear Medicine, 56, 714-720.

https://doi.org/10.2967/jnumed.114.152421

[36] Amini, N., Nakao, R., Schou, M. and Halldin, C. (2013) Identification of PET Radiometabolites by Cytochrome P450, UHPLC/Q-ToF-MS and Fast Radio-LC: Applied to the PET Radioligands $\left[{ }^{11} \mathrm{C}\right]$ Flumazenil, $\left[{ }^{18} \mathrm{~F}\right] \mathrm{FE}-\mathrm{PE} 2 \mathrm{I}$, and $\left[{ }^{11} \mathrm{C}\right] \mathrm{PBR} 28$. Analytical and Bioanalytical Chemistry, 405, 1303-1310. https://doi.org/10.1007/s00216-012-6541-2

[37] Varrone, A., Tóth, M., Steiger, C., Takano, A., Guilloteau, D., Ichise, M., et al. (2011) Kinetic Analysis and Quantification of the Dopamine Transporter in the Nonhuman Primate Brain with ${ }^{11} \mathrm{C}-\mathrm{PE} 2 \mathrm{I}$ and ${ }^{18} \mathrm{~F}-\mathrm{FE}-\mathrm{PE} 2 \mathrm{I}$. Journal of Nuclear Medicine, 52, 132-139. https://doi.org/10.2967/jnumed.110.077651

[38] De Graaf, I.A.M., Van Meijeren, C.E., Pektaş, F. and Koster, H.J. (2002) Comparison of in Vitro Preparations for Semi-Quantitative Prediction of in Vivo Drug Metabolism. Drug Metabolism \& Disposition, 30, 1129-1136. https://doi.org/10.1124/dmd.30.10.1129 